\title{
Síndrome de Brown inflamatória: relato de caso
}

\author{
Inflammatory Brown syndrome: case report
}

Leonardo Guedes Candido Marculino ${ }^{1}$, Thais Zamudio Igami ${ }^{2}$, Andressa Aydar Quadrado ${ }^{1}$, Mauro Goldchmit ${ }^{1,2}$

\section{RESUMO}

A síndrome de Brown enquadra-se no grupo de estrabismos restritivos e é causada pela limitação de movimento do tendão do oblíquo superior através da tróclea. Caracteriza-se por ortotropia ou hipotropia na primária do olhar, limitação ou ausência de elevação em adução, frequente depressão do olho em adução com anisotropia em $\vee$ e dução passiva positiva na elevação em adução. Denomina-se síndrome de Brown inflamatória, o distúrbio secundário, que tem como principais causas inflamações locais na órbita e doenças inflamatórias como artrite reumatóide e tenossinovite. Apresentamos o caso de um paciente de 44 anos, do sexo masculino, com queixa de diplopia binocular vertical à dextroversão recorrente. Diagnosticado como síndrome de Brown inflamatória sem etiologia definida, confirmada por ressonância nuclear magnética e com resolução espontânea.

Descritores: Inflamação; Transtornos da motilidade ocular; Músculos oculomotores; Sindrome; Visão binocular; Tendões/cirurgia; Estrabismo; Espectroscopia de ressonância magnética; Diplopia; Relatos de casos; Humano; Adulto; Masculino

\begin{abstract}
Brown syndrome fits the group of restrictive strabismus and is caused by a movement limitation of the superior oblique tendon through the trochlea. It is characterized by parallelism in the primary gaze position, limitation or absence of elevation in adduction, frequent depression of the eye in adduction with anisotropy in V-pattern and positive passive duction in the elevation in adduction. It is called inflammatory Brown syndrome, a secondary disorder, which main causes are local inflammation in the orbit and inflammatory diseases such as rheumatoid arthritis and tenosynovitis. A case of a 44 year-old patient, male, complaining of binocularvertical diplopia due to recurring dextroversion is reported. It was diagnosed as inflammatory Brown syndrome of uncertain etiology, confirmed by magnetic nuclear resonance, and with spontaneous resolution.
\end{abstract}

Keywords: Inflammation; Ocular motility disorders; Oculomotor muscles; Syndrome; Vision, binocular;Tendons/surgery; Strabismus; Magneticresonance spectroscopy; Diplopia; Case reports; Human; Adult; Male

\section{INTRODUÇÃO}

A síndrome de Brown foi descrita por Harold Brown em 1950 e enquadra-se no grupo de estrabismos restritivos ${ }^{(1-4)}$ É causada pela limitação de movimento do tendão do oblíquo superior através da tróclea, que ocorre por encurtamento do tendão ou inextensibilidade da bainha. Caracterizase por ortotropia ou hipotropia na posição primária do olhar, limitação ou ausência de elevação em adução, frequente depressão do olho em adução com anisotropia em $V$ e dução passiva positiva na elevação em adução ${ }^{(3-5)}$.

No princípio, Brown justificava a restrição do movimento pela perda da força do músculo oblíquo inferior, mas sabe-se, por estudos eletromiográficos, que a função deste está preservada(5).

Possui incidência de 1/500 estrábicos ${ }^{(3)}$, sem preferência de sexo e acometimento ocular unilateral em sua maioria ${ }^{(3-4)}$. Pode ser classificada como congênita ou adquirida (ou secundária). Esta forma engloba os casos consecutivos a traumatismos, pregueamento cirúrgico do tendão (iatrogênica) ou por processo inflamatório adjacente. Neste caso, denomina-se síndrome de Brown inflamatória, que pode ser ocasionada por alguma doença reumatológica, pansinusite, esclerite ou idio-

Trabalho realizado no Instituto Cema, Hospital Cema - São Paulo (SP), Brasil.

Médico, Departamento de Oftalmologia, Hospital Cema - São Paulo (SP), Brasil.

Médico, Departamento de Oftalmologia, Irmandade Santa Casa de Misericórdia de São Paulo - ISCMSP - São Paulo (SP), Brasil.

Endereço para correspondência: Leonardo Guedes Candido Marculino. Rua Eunice Weaver, 215 - São Paulo (SP) - CEP 03333-060

Weaver, 215 - São Paulo (SP) - CEP

Recebido para publicação em 11.04.2009

Última versão recebida em 13.11.2009

Aprovação em 17.11.2009 pática ${ }^{(3-4)}$. Inflamações locais na órbita e doenças inflamatórias como artrite reumatóide e tenossinovite são as principais causas desse subtipo ${ }^{(5-6)}$. O tratamento para estes casos inclui corticóides orais ou injeções de corticóide próximas à tróclea e abordagem da doença de base. A intervenção cirúrgica está indicada, caso haja posição viciosa da cabeça (desvio torcional) ou desvio em posição primária do olhar ${ }^{(3-4,7)}$. Apresentaremos um caso de síndrome de Brown inflamatória confirmada clínica e radiologicamente.

\section{RELATO DE CASO}

Paciente de 44 anos, do sexo masculino, professor, natural e procedente do município de São Paulo, queixava-se de diplopia binocular vertical à dextroversão há sete dias, com duração de um dia, e que melhorava com a elevação do mento. No momento da consulta, encontrava-se assintomático.

Referiu sintoma semelhante há quatro anos, com duração de três dias. Nega casos de estrabismo na família.

Ao exame oftalmológico, apresentava acuidade visual de 1,0/em ambos os olhos sem correção. Sua refratometria dinâmica indicava $+0,25$ dioptrias esféricas em ambos os olhos. Não apresentava anormalidades à biomicroscopia e fundoscopia. $\mathrm{Na}$ avaliação da motilidade ocular extrínseca, observou-se ortotropia em todas as posições do olhar, sem alterações no exame de duçõese versões. Adotou-se conduta expectante. O paciente foi orientado a retornar, caso o quadro clínico se repetisse.

Após quatro meses, o paciente retornou referindo diplopia binocular há um dia. O exame da motricidade ocular apresentava-se com ortotropia na posição primária do olhar e hipotropia esquerda à dextroversão (Figura 1 A), com limitação de elevação e adução do olho esquerdo de -4 (Figura 1 B). Realizada a manobra de tentativa de elevação do globo ocular com 

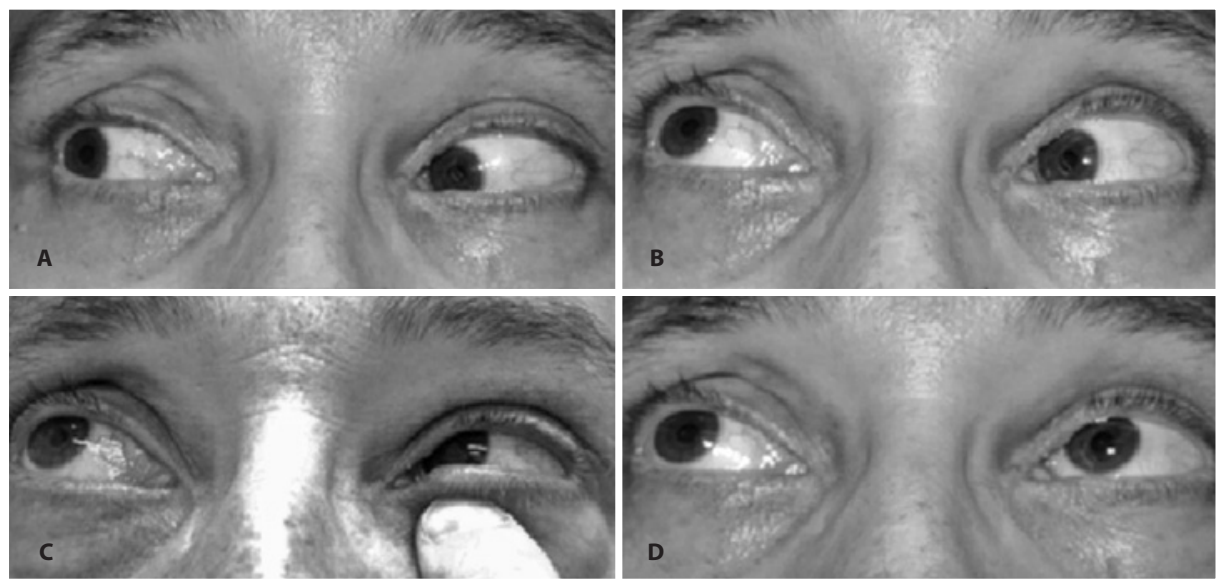

Figura 1. A) Hipotropia esquerda à dextroversão; B) Limitação de elevação em adução do olho esquerdo de -4;C) Manobra de tentativa de elevação do globo ocular com dedo indicador; D) Normalização das versões.

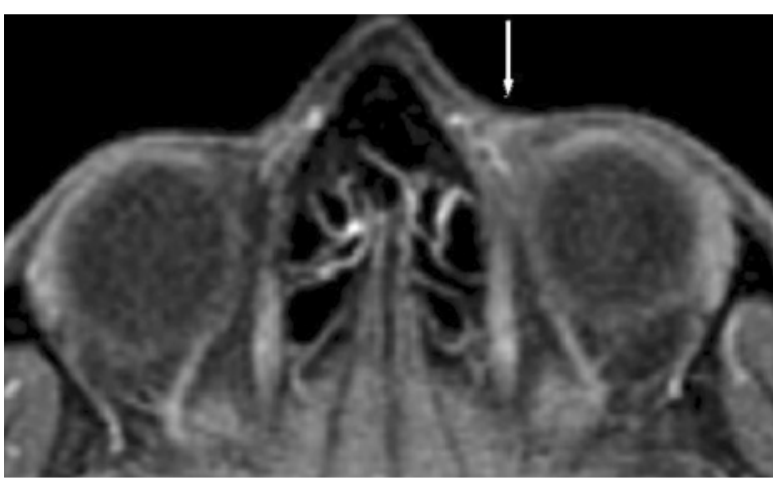

Figura 2. Seta destacando o hipersinal na região da tróclea sugerindo processo inflamatóriolocal.

dedo indicador (Figura 1 C), o que permitiu a normalização das versões (Figura 1 D). Apresentava dor à palpação da região da tróclea.

Foram solicitados exames laboratoriais (hemograma completo, fator reumatóide, fator antinuclear, anti-RO/SS-A, antiLA/SS-B, velocidade de hemossedimentação, proteína C-reativa, uréia, creatinina, aspartatoaminotransferase, alanina transaminase, fosfatase alcalina, gama-glutamiltranspeptidase, exame qualitativo da urina, análise do líquido sinovial, radiografia das mãos, anticorpo antipeptídiocitrulinado, sorologia para hepatite B e C, ANCA C e P, antígeno HLA-B27, comple- mento total e componentes, urina I e PPD) para pesquisa de doenças reumatológicas e infecciosas, estando todos dentro da normalidade. O exame de ressonância nuclear magnética (RNM) de órbita demonstrou hipersinal na região da tróclea (Figura 2) sugerindo processo inflamatório local.

\section{DISCUSSÃO}

A síndrome de Brown inflamatória é um tipo raro de estrabismo restritivo adquirido, no qual ocorre o acometimento do tendão do músculo oblíquo inferior de provável causa reumatológica subjacente. A manobra de tentativa de elevação mecânica do bulbo ocular com o dedo resultou em normalização da dução, o que sugere o diagnóstico de síndrome de Brown adquirida, quadro este frequentemente associado a condições inflamatórias ${ }^{(4-5)}$. 0 quadro clínico autolimitado e recidivante, associado à alteração encontrada no exame de RNM confirmou o diagnóstico.

A resolução espontânea da síndrome de Brown adquirida não traumática ocorre em aproximadamente $16 \%$ dos pacientes ${ }^{(4,6,8)}$ contraindicando o tratamento cirúrgico. O caso apresentado evoluiu com resolução espontânea, mesmo sem administração de corticóides, como em alguns relatos encontrados ${ }^{(9-10)}$.

\section{REFERÊNCIAS}

1. Hargrove RN, Fleming JC, Kerr NC. Brown's syndrome in the absence of an intact superior oblique muscle. J AAPOS. 2004:8(5):507-8.

2. Parks, MM, Brown M. Superior oblique tendon sheath syndrome of Brown. Am J Ophthalmol. 1975;79(1):82-6.

3. Souza-Dias, CR. Estrabismo. São Paulo: Rocca:1993.

4. Wright KW. Brown syndrome [Internet]. Update 2010 Feb 8. [cited 2008 Dec 13]. Available from: http://emedicine.medscape.com/article/1199301-overview

5. Booth-Mason S, Kyle GM, Rossor M, Bradbury P. Acquired Brown's syndrome: an unusual cause. Br J Ophthalmol. 1985;69(10):791-4.

6. Wright KW. Brown's syndrome: diagnosis and management. Trans Am Ophthalmo Soc. 97 1999;97:1023-109.

7. Hadjadj E, Conrath J, Ridings B, Denis D. [Brown syndrome: current status]. J Fr Ophtalmol.1998:21(4):276-82. French.

8. Mühlendyck H. [Jaensch-Brown syndrome-etiology and surgical procedure]. Klin Monbl Augenheilkd. 1996;208(1):37-47. German.

9. Maggi R, Maggi C. Tendon surgery in Brown's syndrome. J Pediatr Ophthalmol Strabismus. 2002;39(1):33-8.

10. Kirsch D, Lima CAVC, Yamamoto MM, Andrade EP, Perez MF. Síndrome de Brown bilateral associada com hipermobilidade articular benigna: relato de caso. Arq Brás Oftalmol. 2007;70(2):360-2

Ao enviar um artigo para publicacão, leila AUENTAMENTE as instrucōes para autores, constante no final de cada fascículo. 